\title{
Performance of ALICE Silicon Tracking Detectors
}

\author{
Stefania BUFALINO* \\ INFN - Sezione di Torino \\ E-mail: stefania.bufalinodcern.ch \\ for the ALICE Collaboration
}

The ALICE detector was designed to track and identify particles in a wide transverse momentum range, from $\sim 100 \mathrm{MeV} / \mathrm{c}$ to more than $100 \mathrm{GeV} / \mathrm{c}$. The Inner Tracking System (ITS) of the ALICE experiment consists of six cylindrical layers of silicon detectors. Each layer has hermetic structure and it is coaxial with the beam pipe. The ITS covers the pseudorapidity range $|\eta| \leq 0.9$ and the distance from the nominal beam line ranges from $3.9 \mathrm{~cm}$ for the innermost layer up to 43 $\mathrm{cm}$ for the outermost. The two innermost layers are made of Silicon Pixel Detectors (SPD), the two central layers of Silicon Drift Detectors (SDD) and the two outermost layers of double sided Silicon Strip Detectors (SSD). In this proceeding we present the ITS performance for tracking, interaction vertex reconstruction and particle identification capability. Precise tracking is required to reconstruct and separate the primary vertex and secondary vertices from heavy-flavour meson decays: one example is reported.

The 21st International Workshop on Vertex Detectors

16-21 September 2012

Jeju, Korea

${ }^{*}$ Speaker. 


\section{Introduction}

The ALICE detector is composed of two main sections: a central barrel, covering the full azimuth in the pseudo-rapidity region $|\eta| \leq 0.9$ and a forward $(2.5 \leq \eta \leq 4)$ muon arm [2]. The ITS (Inner Tracking System) is a cylindrically-shaped silicon tracker in the central barrel that surrounds the interaction region. Its main task is to provide precise track and vertex reconstruction close to the interaction point. In particular, the ITS was designed to improve the position, angle, and momentum resolution for tracks reconstructed in the Time Projection Chamber (TPC, inner radius 80 $\mathrm{cm}$ ), to identify the secondary vertices from the decay of hyperons and heavy flavoured hadrons, to reconstruct the interaction vertex with a resolution better than $100 \mu \mathrm{m}$, and to recover particles that are missed by the TPC due to acceptance limitations. In this proceeding we present the ITS performance for tracking, primary vertex reconstruction, particle identification capability and secondary vertices identification. We start by describing the ITS layout in Section 2. To achieve the design performance and to tune MC simulations in order to reproduce as realistically as possible the detector response and geometry, alignment and a good knowledge of the material budget are required. These are addressed in Section 3. The actual tracking and vertexing performance is reported in the same section. The ITS capability for particle identification at low momentum is described in Section 4 together with the result of physics analyses based on the precise track and vertex reconstruction provided by the ITS.

\section{ALICE Inner Tracking System Layout}

The Inner Tracking System (ITS) is the innermost detector of the ALICE experiment [1,2] at the CERN Large Hadron Collider. The ITS consists of six cylindrical layers of position-sensitive

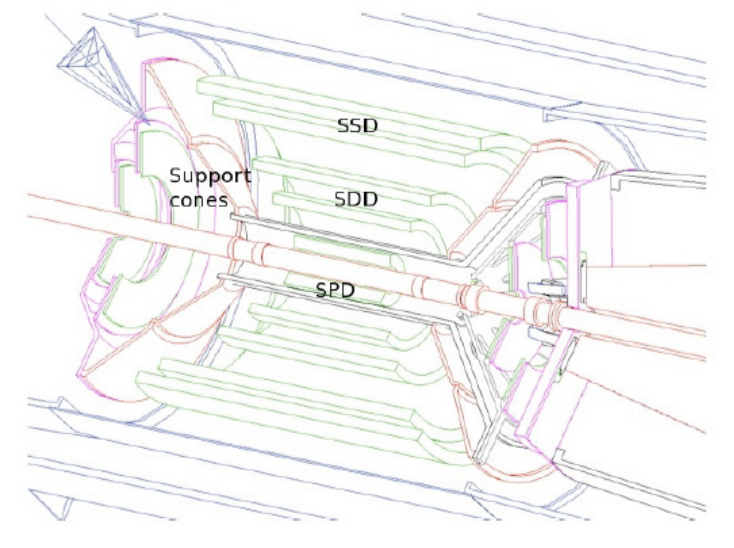

Figure 1: Layout of the Inner Tracking Sistem of the ALICE experiment.

detectors, covering the central rapidity region $(|\eta| \leq 0.9)$ for vertices located within $10 \mathrm{~cm}$ along the beam direction $(z)$. The main parameters of the various layers of the ITS are summarized in Table 1. Three different silicon detector technologies have been adopted for the ITS: pixels for the two innermost layers at radii of 3.9 and $7.6 \mathrm{~cm}$ from the beamline (the Silicon Pixel Detector, SPD), silicon drifts in the two intermediate layers at 15 and $24 \mathrm{~cm}$ (Silicon Drift Detector, 
Table 1: Main parameters of the ITS detectors.

\begin{tabular}{ccccccccr}
\hline Layer & Type & $r(\mathrm{~cm})$ & $\pm z(\mathrm{~cm})$ & Area $\left(\mathrm{m}^{2}\right)$ & Ladders & Ladders/stave & Det./ladder & Tot. channels \\
\hline 1 & pixel & 3.9 & 16.5 & 0.09 & 80 & 4 & 1 & 5242880 \\
2 & pixel & 7 & 16.5 & 0.18 & 160 & 4 & 1 & 10485760 \\
3 & drift & 14.9 & 22.2 & 0.42 & 14 & - & 6 & 43008 \\
4 & drift & 23.8 & 29.7 & 0.89 & 22 & - & 8 & 90112 \\
5 & strip & 39.1 & 45.1 & 2.28 & 34 & - & 23 & 1201152 \\
6 & strip & 43.6 & 50.8 & 2.88 & 38 & - & 26 & 1517568 \\
\hline \multicolumn{7}{c}{ Total area $=6.74 \mathrm{~m}^{2}$} \\
\hline
\end{tabular}

SDD) and double-sided strips for the two outermost ones at 38 and $43 \mathrm{~cm}$ (Silicon Strip Detector, SSD). The geometrical layout of the ITS layers, as implemented in the ALICE simulation and reconstruction software framework (AliRoot [3]), is shown in Fig. 1. The number, position and segmentation of the layers and the chosen technology were optimized for efficient track finding and high impact-parameter resolution in the severe high-multiplicity environment expected for central $\mathrm{Pb}-\mathrm{Pb}$ collisions at LHC energy. In particular, the outer radius is determined by the necessity to match tracks with those reconstructed in the other main tracking device, the large volume Time Projection Chamber (TPC), and the inner radius is the minimum allowed by the radius of the beam vacuum tube. The high particle density expected in heavy-ion collisions at the LHC (up to 50 particles per $\mathrm{cm}^{2}$ for the layer closest to the interaction point) and the required impact parameter resolution (better than $100 \mu \mathrm{m}$ for tracks with $\mathrm{p}_{T}>1 \mathrm{GeV} / \mathrm{c}$ ) have dictated the choice of Silicon Pixel Detectors (SPD) for the two innermost layers. The SPD has binary readout and also provides a prompt (Fast- OR) trigger signal contributing to the L0 trigger of the experiment. The SDD and SSD have analogue readout and therefore can be used for particle identification via $\mathrm{dE} / \mathrm{dx}$ measurement in the non-relativistic $\left(1 / \beta^{2}\right)$ region. This feature gives the ITS stand-alone capability as a low $\mathrm{p}_{T}$ particle spectrometer. The SPD, SDD and SSD comprise each 240, 260 and 1698 detector modules, respectively. The innermost SPD layer has a more extended pseudorapidity coverage $(|\eta| \leq 1.98)$ to provide a larger acceptance for the measurement of charged-particles multiplicity. A detailed description of the ITS detectors can be found in [2].

\section{ITS performance for Vertex and Track reconstruction}

\subsection{Alignment and SDD calibration}

The target of the alignment procedure is to measure the detector geometry with great accuracy in order to limit the error on relevant measured quantities to $20 \%$ of the nominal values (obtained by detailed MC simulations and compared to data as in Fig.2). In the case of ITS, this is a challenging task due to the high intrinsic precision of the sensors and to the large number of degrees of freedom (more than 13000). The alignment procedure uses the optical and mechanical survey measurements as a starting point for the realignment. Survey information about the sensor positions on ladders (linear assemblies of sensors at the same azimuthal angle) was available for both SSD and SDD. 
Positions of the SSD ladders with respect to the supporting cones have also been measured. The final alignment precision can be reached using reconstructed tracks. Two different algorithms for the minimization of the point-to-track residuals are used to determine the most probable position of the modules in the ALICE reference frame: Millepede [5] where a global fit to all residuals is performed, extracting all the alignment parameters simultaneously and an iterative module-by-module approach [4] where a (local) minimization for each single module is performed and the correlations between modules is accounted by iterating the procedure until convergence is reached. The current strategy includes the use of both cosmic ray and proton-proton collision tracks, with and without magnetic field. The results achieved with cosmic-ray data collected in 2008 are described in $[6,7,8]$. About $10^{5}$ tracks from cosmic-ray events with zero magnetic field were considered for the ITS alignment. Cosmic-ray tracks have a dominant vertical component and the sides of the barrel layers have limited statistics. A refined alignment using about $2 \cdot 10^{7} \mathrm{p}-\mathrm{p}$ collision tracks at $7 \mathrm{TeV}$ and a few $10^{4}$ cosmic ray tracks collected in 2009-2010 was performed and used in the extraction of the physics results of ALICE. The following three observables are used to check the quality of the alignment: the track-to-point residuals, the track-to-point distance for clusters in the overlapping regions between modules of the same layer (extra-clusters) and, with cosmic-ray tracks only, the top half-track to bottom half-track matching at the plane $y=0\left(\left.\Delta x y\right|_{y=0}\right)$. The latter provides a direct measurement of the resolution on the track transverse impact parameter $\mathrm{d}_{0}(\mathrm{r} \phi)$, namely $\sigma_{\left.\Delta x y\right|_{y=0}}\left(p_{T}\right)=\sqrt{2} \sigma_{d_{0}(r \phi)}\left(p_{T}\right)$.

The first step of the alignment procedure was the validation of the SSD survey. This was done using extra clusters over the full azimuthal angle and as a function of the transverse momentum has been performed. The widths of the point-to-track distributions of extra clusters confirm that the residual misalignment is compatible with the nominal precision of the survey measurements (i.e. less than $5 \mu \mathrm{m}$ RMS for modules on the same ladder and less than $20 \mu \mathrm{m}$ RMS for modules on different ladders), as already verified in [6] for the top and bottom regions of the detector.

The SPD modules were then aligned with Millepede, keeping the SSD modules fixed and using cosmic ray tracks and $\mathrm{p}-\mathrm{p}$ collision tracks with magnetic fields $\mathrm{B}=0, \mathrm{~B}=+0.5 \mathrm{~T}$ and $\mathrm{B}=-0.5 \mathrm{~T}$ at the same time. With respect to Ref. [6] a better alignment for SPD was achieved. The mean values of local residual distributions for SPD modules on both layer 1 and layer 2 are of the order of a few microns, at high $\mathrm{p}_{T}$ over the full azimuthal angle (except for a few modules with poor or null statistics due to hardware malfunctions). We verified, analyzing the $\mathrm{p}-\mathrm{p}$ data at $7 \mathrm{TeV}$, that the point-to-track distributions of extra clusters are compatible with the MC simulation in which a residual misalignment of about $8 \mu \mathrm{m}$ RMS for SPD and the nominal precision of the survey measurements for the SSD are taken into account. The results are shown in Fig. 2 and discussed in details in [9].

As far as the SDD are concerned, the local x coordinate is calculated from the measured drift time according to the formula $x_{l o c}=\left(t-t_{0}\right) v_{d r i f t}$. Two complementary methods have been developed in order to obtain a first estimate of the $t_{0}$ parameter. The first, and simpler, method consists in extracting the $t_{0}$ from the minimum measured drift time on a large statistics of reconstructed SDD points. The second method measures the $t_{0}$ from the distributions of residuals along the drift direction $\left(x_{l o c}\right)$ between tracks fitted in SPD and SSD layers and the corresponding points reconstructed in the SDD. This second procedure has the advantage of requiring smaller statistics, because it profits from all the reconstructed tracks, with the drawback of relying on SDD calibration param- 
eters (the drift velocity and possibly the correction maps). $\mathrm{V}_{\text {drift }}$ is obtained by means of MOS charge injectors integrated on the detector surface. Corrections based on point-to-track residual must be applied to the measured value for $v_{\text {drift }}$ for modules with malfunctioning injectors $(30 \%$ of the total) and to account for systematic effects.
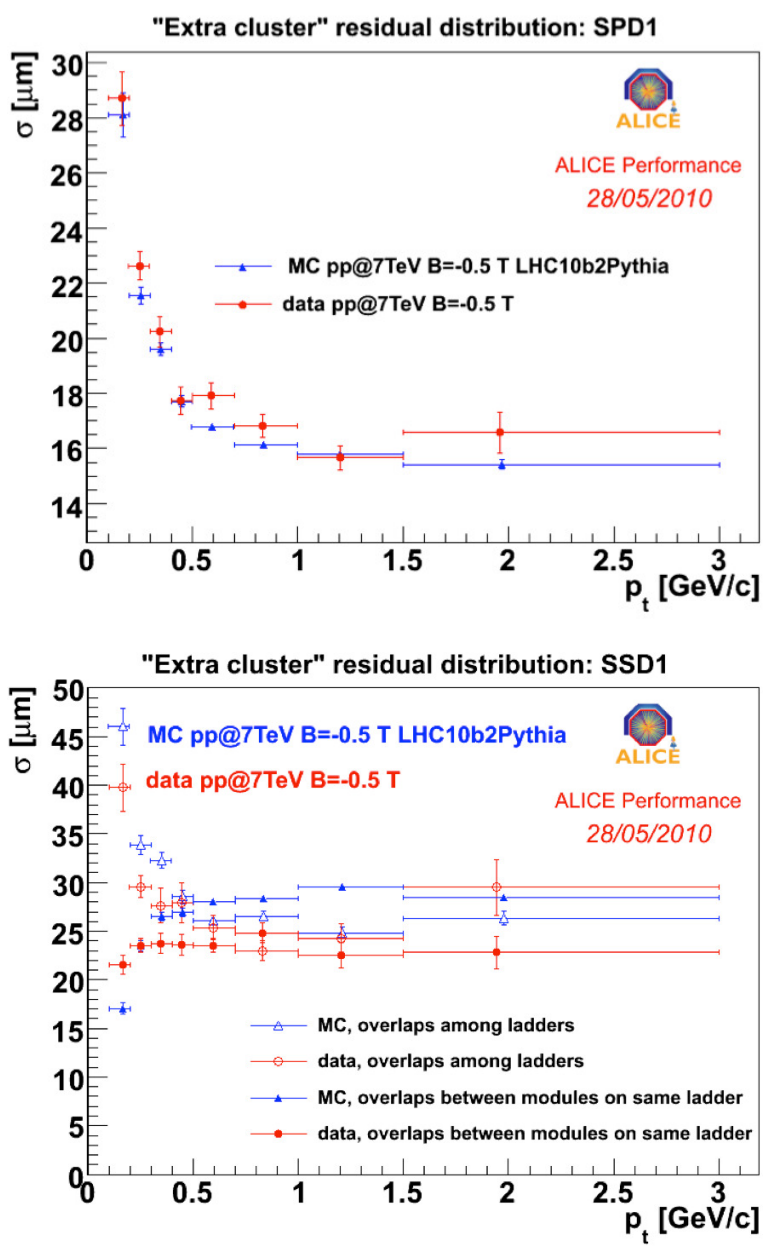

Figure 2: Extra cluster residual distribution on SPD1 (upper) and SSD1 (bottom) both for data and MC.

Corrections maps can be extracted from laser laboratory reference measurements and from $\mathrm{p}-\mathrm{p}$ data. The rightmost panel of the left side of Fig. 3 shows the effectiveness of the correction for the drift field non-uniformity extracted from the track-to-point residuals. The corrections are able also to correct possible defects in the integrated voltage divider resulting in a non-uniform drift field. After having applied corrections to the local residual profiles as a function of the drift speed, the alignment of the SDD modules was also performed. A special implementation in Millepede of the drift time initial value and the drift speed as extra alignment parameters has been used. The width of the local residual distributions for SDD modules in layer 3 and 4 is shown in Fig. 3 (right panel) as a function of the transverse momentum, before and after corrections. A final uncertainty (calibration 

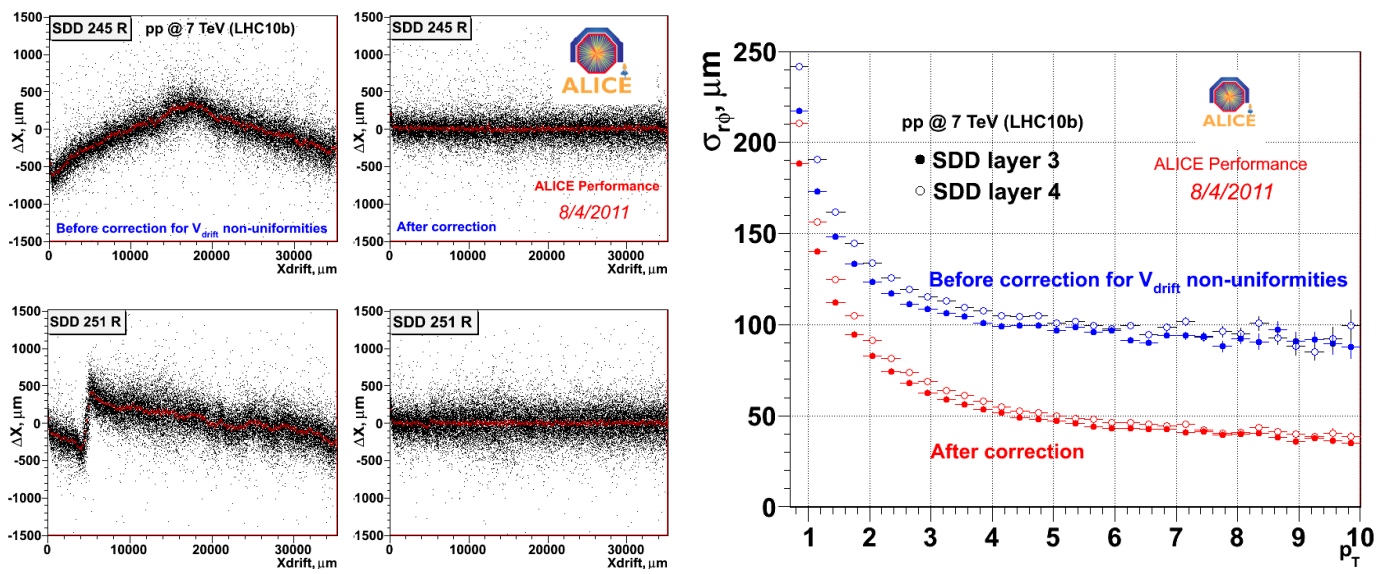

Figure 3: Left: Track-to-point residuals along local $\mathrm{X}$ (drift direction) as a function of local $\mathrm{X}$ for two SDD modules before and after the correction for non uniformity of the drift field. Right: width of the residual distribution as a function of transverse momentum, before and after correction.

+ alignment) of about $30-35 \mu \mathrm{m}$ in the $r \phi$ plane (comparable with the nominal resolution of the detector) was achieved for high momentum tracks.

\subsection{Vertex determination and pile-up tagging}

The reconstruction of the interaction vertex position (vertexing in the following) is one of the key tasks that the ITS has to accomplish. This is currently done by means of three different methods depending on the reconstruction stage (online, local reconstruction, final reconstruction after tracking) and the colliding system ( $\mathrm{p}-\mathrm{p}$ and $\mathrm{Pb}-\mathrm{Pb})$.

Two methods are based only on the SPD local reconstruction, i.e. only on pairs of reconstructed points in the 2 SPD layers (tracklets). They are the VertexerSPDz and the VertexerSPD3D. The VertexerSPDz provides the measurement of the $\mathrm{z}$ coordinate (along the beam axis) of the interaction point, using the average location of the collision region in the transverse plane. The VertexerSPD3D provides a three dimensional measurement of the interaction point and the vertices measured online in this way, are selected according to a set of quality cuts and then averaged. The resulting Mean Vertex is stored in the Offline Conditions DataBase (OCDB): it represents a first determination of the luminous region. During the offline reconstruction, the approach is different for $p p$ and $\mathrm{Pb}-\mathrm{Pb}$ data. In the $\mathrm{pp}$ case, a first 3D vertex reconstruction with the VertexerSPD3D algorithm is attempted for all the events. If the vertexing fails, typically because of the low number of high momentum particles in the SPD acceptance, the reconstruction of the $\mathrm{z}$ coordinate of the collision point is done with the VertexerSPDz algorithm, which has high efficiency and a low CPU impact. For $\mathrm{Pb}-\mathrm{Pb}$ data, the VertexerSPD3D algorithm is too slow to be used for the offline reconstruction of all the collected events, due to the high combinatorial background: the first vertex determination is always done with the VertexerSPDz algorithm and the Mean Vertex knowledge.

A third method for precise determination of the primary interaction vertex can be obtained using tracks instead of tracklets and is used both for $\mathrm{p}-\mathrm{p}$ and $\mathrm{Pb}-\mathrm{Pb}$ data in the final recontruction phase after tracking. The VertexerTracks algorithm is a three iterations procedure that allows to obtain 
a precise measurement of the primary vertex, using tracks reconstructed in the whole ALICE central barrel with the ITS and the Time Projection Chamber (TPC). The first iteration consists in a preselection of tracks and in a rough preliminary vertex estimate used as a starting point for the reconstruction. The two subsequent iteration passes consist of three steps: TRACK SELECTION to reject displaced tracks, VERTEX FINDING to perform a first estimate of the vertex position and VERTEX FITTING in which the tracks are propagated to the position estimated in the previous step and the optimal estimate of the vertex position and its covariance matrix is computed. In $\mathrm{Pb}-\mathrm{Pb}$ collisions the track multiplicity per event is so high that allows the use of the so called "half-event" method to evaluate the resolution on the vertex position. In this algorithm, for each event, the track sample is randomly divided in two, and a primary vertex is reconstructed for each of the two sub-samples. The resolution is thus extracted from the $\sigma$ of the distribution of the residuals between the two vertices. In Fig. 4 the vertex resolution is plotted in a log-log scale as a function of the tracklet multiplicity for the transverse (x) and longitudinal (z) coordinates. The fit to the points is performed using the function repoted in black on the figure and considering the data points with an half event tracklet multiplicity greater than 10 where we are less dominated by the residual background. The fit is used to extrapolate the value of the resolution at the multiplicity we measured for events in the $0-5 \%$ centrality class (orange box). The corresponding vertex resolution turns out to be better than $10 \mu \mathrm{m}$ both for $\mathrm{x}$ and $\mathrm{z}$ coordinates for multiplicities above $10^{3}$.

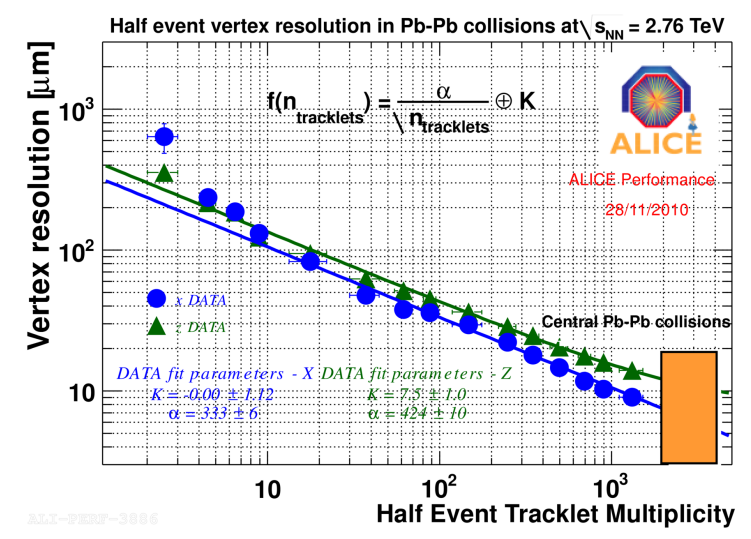

Figure 4: Vertex resolution estimated with the half-event technique in $\mathrm{Pb}-\mathrm{Pb}$ collisions at $\sqrt{s}=2.76 \mathrm{TeV}$.

Since the readout time of the SPD is $100 \mathrm{~ns}$, up to 4 independent bunch crossings are expected to occur in one SPD event. Due to its fast response and very low occupancy in pp collisions ( $\sim 10^{-4}$ even for high multiplicity events), the SPD layers can be used to check for the presence of multiple collision points (pile-up vertices). A pile-up tagging algorithm has been developed for both the SPD based vertex finding methods. In both cases, when multiple collisions occur in a 100 ns window, the vertex position corresponds to the interaction with the highest multiplicity. These vertices are referred to as the main vertices. A search for other possible interaction points is done essentially by running again the vertexing algorithm excluding the tracklets coming from a fiducial region centered around the main vertex. A pile-up candidate is accepted if there is a minimum number of tracklets $\left(N_{\min }\right)$ pointing to it and if its separation from the main vertex along the beam axis $(\Delta \mathrm{z})$ is large enough to avoid the reconstruction of a false positive, that is a fake vertex built 
with tracklets originating from the main collision point. The distribution of $\Delta \mathrm{z}$ for candidates pileup vertices is shown in Fig. 5. For two different selections on the number of tracklets it can be seen that the peak at $\Delta \mathrm{z}=0$ is due to tails of tracklets belonging to the main vertex, but reconstructed as a second vertex (false positives) and it is cut by a selection on the number of contributors. The performance of the pile-up tagging algorithm has been studied by means of dedicated Monte Carlo simulations, in which different pp events were simulated, reconstructed and using different MC event generators. The actual tagging efficiency depends on the available tracklets to build a candidate vertex, hence on the charged particle multiplicity at mid rapidity.

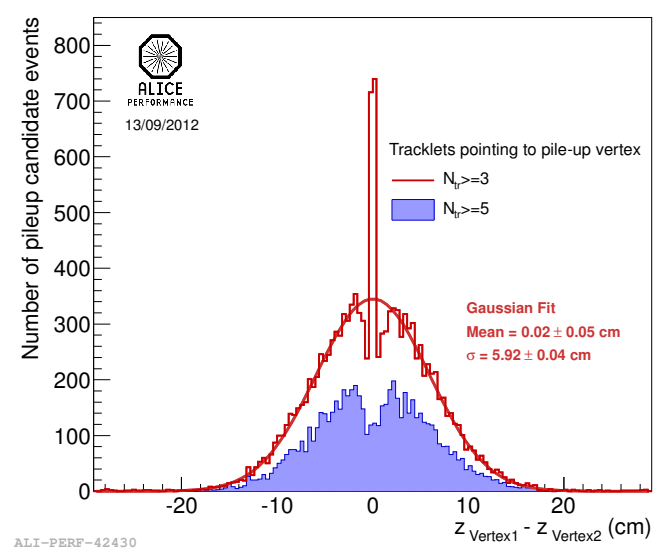

Figure 5: Distribution of the difference between the $\mathrm{z}$ coordinates of the main and pile-up vertices reconstructed with the SPD.

\subsection{Tracking}

Full track reconstruction is performed via a Kalman filtering algorithm [2]. Charged tracks are parametrized by 5 parameters describing a helix. In the Kalman filtering approach the track trajectory is only locally a helix: the track parameters are updated at each point along the track trajectory allowing the possibility to account for deviations due to the interaction with the material (energy loss and multiple scattering). Starting from some initial approximation for the track parameters, points to be added to the track are searched inside a road determined by the uncertainties on the track extrapolation at a given plane and on the cluster position at the same plane. An additional uncertainty on the ITS cluster position has been added to the intrinsic resolution to account for residual misalignment. Track reconstruction is performed in the following steps. The first one is the computation of the primary vertex using SPD tracklets. Track reconstruction then starts in the TPC, using the outermost pad rows and the computed primary vertex position as seed. Track seeds are prolonged in the inward direction until the TPC inner radius is reached. The track reconstruction continues in the ITS after matching TPC reconstructed tracks to the SSD layers and following them through the SDD till the innermost SPD layer. The next step is track back-propagation, to the outermost layer of the ITS, to the outermost radius of the TPC and, after the extrapolation and the track finnding in the TRD, to the outer detectors (TOF, HMPID, PHOS, EMCal) for Particle IDentification (PID). A refit step is then performed: reconstructed tracks are re-fitted inward in 
TRD, TPC, ITS and are propagated to the primary vertex reconstructed in the first step. Finally the primary vertex is recalculated with optimal resolution using reconstructed tracks. To extend the $p_{T}$ acceptance down to $\sim 100 \mathrm{MeV} / \mathrm{c}$ and to recover tracks not reconstructed in the TPC, an ITSstandalone tracking has been developed to reconstruct tracks in the ITS detector alone. In Fig.6 (left) the prolongation efficiency between TPC and ITS is reported as a function of transverse momentum. It is above $95 \%$ and almost costant in the whole momentum range, if tracks with at least two points in the ITS are considered. It drops down to $85 \%$ if the further request of one point in the SPD is applied, due to the presence of inactive modules. MC simulations reproduce the efficiency trend observed in data within $2 \%$ in the range $0.2 \leq p_{T} \leq 10 \mathrm{GeV} / \mathrm{c}$.
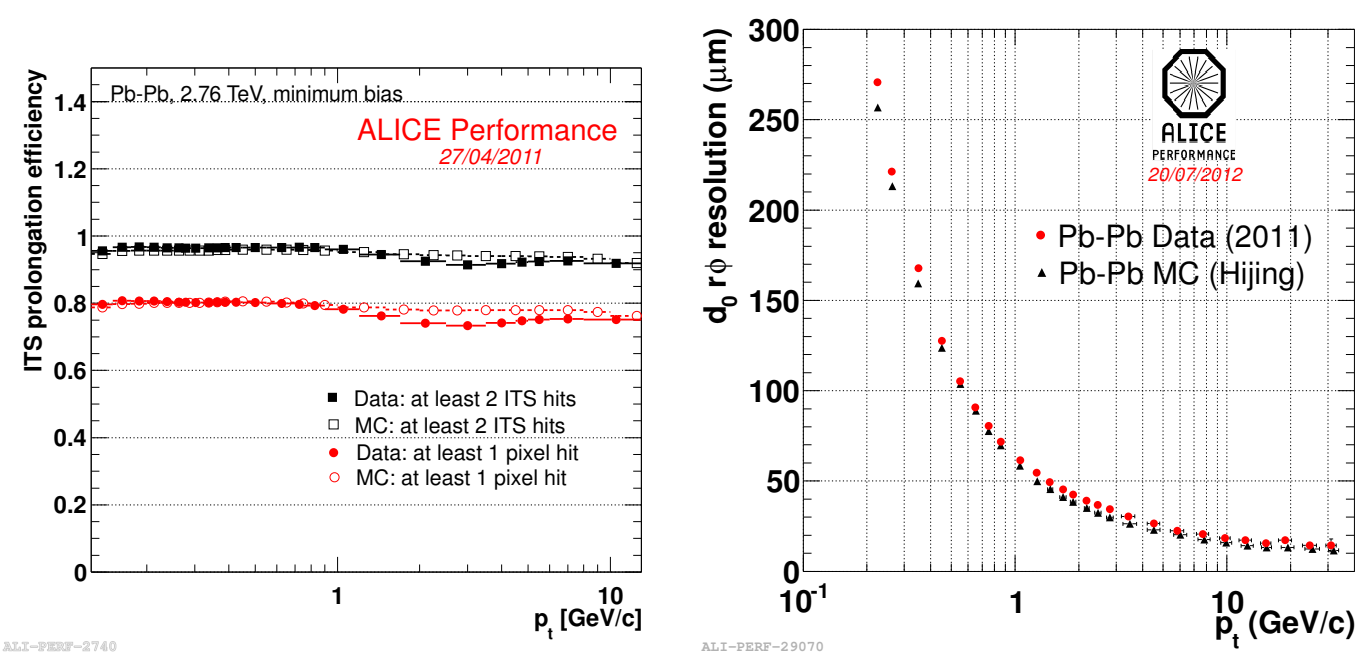

Figure 6: Left: (left) track prolongation efficiency from TPC to ITS as a function of transverse momentum in the case two points in the ITS are required (square markers) and with the further request that at least one point is in the SPD (round markers). Filled markers refers to data from pp collisions at $7 \mathrm{TeV}$ while empty markers refer to MC simulations. Right: transverse impact parameter resolution. Tracks satisfying the standard TPC track quality cuts and with 2 points in the SPD were considered. For each track, the impact parameter was computed with respect to the primary vertex reconstructed without using the same track.

The resolution on the track impact parameter $\left(d_{0}\right)$, computed as the distance of closest approach (DCA) of a given track to the vertex, is a benchmark to define the tracking precision of the system. In Fig. 6 (right) we plot the resolution on the transverse component of the impact parameter for tracks reconstructed in the central barrel and satisfying the standard track quality cuts. In the plot we show results for tracks reconstructed for $\mathrm{Pb}-\mathrm{Pb}$ collisions: the resulting impact parameter resolution is the convolution of the track-position and the primary-vertex resolutions. As shown in the previous section the contribution of the vertex resolution $(\sim 10 \mu \mathrm{m})$ is negligible in this $\mathrm{p}_{T}$ range. The results for $\mathrm{Pb}-\mathrm{Pb}$ collisions (red dots) are compared with a sample of MonteCarlo (MC) minimum bias events (black triangles) generated using the Hijing event generator [12]. Data and MC appear to be in good agreement. 


\section{Particle Identification}

The particle identification in the Inner Tracking System (ITS) is based on the specific energy deposit per unit path length of a particle, which depends for a given momentum only on its charge and rest mass. Thus, the simultaneous measurement of track momentum (or rigidity) and signal amplitude in a sensitive detector volume allows one to identify particles. The measured mean energy deposit of a track is denoted as $\mathrm{d} E / \mathrm{d} x$ hereafter. In practice, it can be described with a parametrization of the well-known Bethe-Bloch formula. Silicon Drift Detectors (SDD) and Silicon Strip Detectors (SSD) provide analogue read-out for up to four samples, which are combined in a truncated mean to abtain a single $\mathrm{d} E / \mathrm{d} x$ value. The $\mathrm{Pb}-\mathrm{Pb}$ results are shown in figure 7 (left). A resolution of $\sigma_{d E / d x} \approx 10-15 \%$ is achieved. The particle identification in the ITS combined with stand-alone tracking allows to identify pions down to a minimum momentum of $p_{T} \approx 100$ $\mathrm{MeV} / \mathrm{c}$ which reduces the systematic error of yield and $\left\langle p_{T}\right\rangle$ measurements due to extrapolation to $p_{T} \sim 0$. The ITS PID capability also provides pion/kaon separation up to $0.5 \mathrm{GeV} / \mathrm{c}$ and pion/proton separation up to about $1 \mathrm{GeV} / \mathrm{c}$.

\section{Physics performance}

The precise measurement of the track impact parameter and the capability to separate the secondary vertices originating from heavy flavour decays are fundamental requirements for the reconstruction of decays of heavy flavour (charm and beauty) particles. The analysis strategy for the study of charmed mesons is based on the invariant mass analysis of fully reconstructed decay topologies originating from displaced vertices. The aim is to correctly assign the tracks belonging to the open charm decays from the full sample of reconstructed tracks. Due to the large combinatorial background, a series of selection criteria has been defined: the cuts are optimized to maximize the statistical significance of each specific decay signal. The particle identification (based on the TOF and TPC detectors signals) is also applied for the track selection, in particular in the high multiplicity environment of $\mathrm{Pb}-\mathrm{Pb}$ collisions. As an example of the good performance achieved with the ITS, the invariant mass distributions of one benchmark channel, namely $D^{0} \rightarrow K^{-} \pi^{+}$, is reported in fig. 7 (right). Precise reconstruction of the primary vertex and of the track trajectory in the vicinity of the vertex are essential prerequisites to perform these analyses: the performance of the ITS is thus crucial.

\section{Summary}

In this paper we have described the performance of the ALICE Inner Tracking System. The quality of the calibration and the alignment is costantly monitored by means of dedicated quality assurance checks during the whole data taking period. $\mathrm{In} \mathrm{Pb}-\mathrm{Pb}$ collisions we reached a resolution of $\approx 60 \mu \mathrm{m}$ on the tracks impact parameter for tracks with $\mathrm{p}_{T}>1 \mathrm{GeV} / \mathrm{c}$. The vertex position and tracklets reconstructed in the SPD were also used to tag the pile-up events in p-p collisions. The ITS is used as a charged particle identification system for low momentum tracks and it has been shown that it covers a fundamental role in the fulfillment of ALICE physics targets like in 

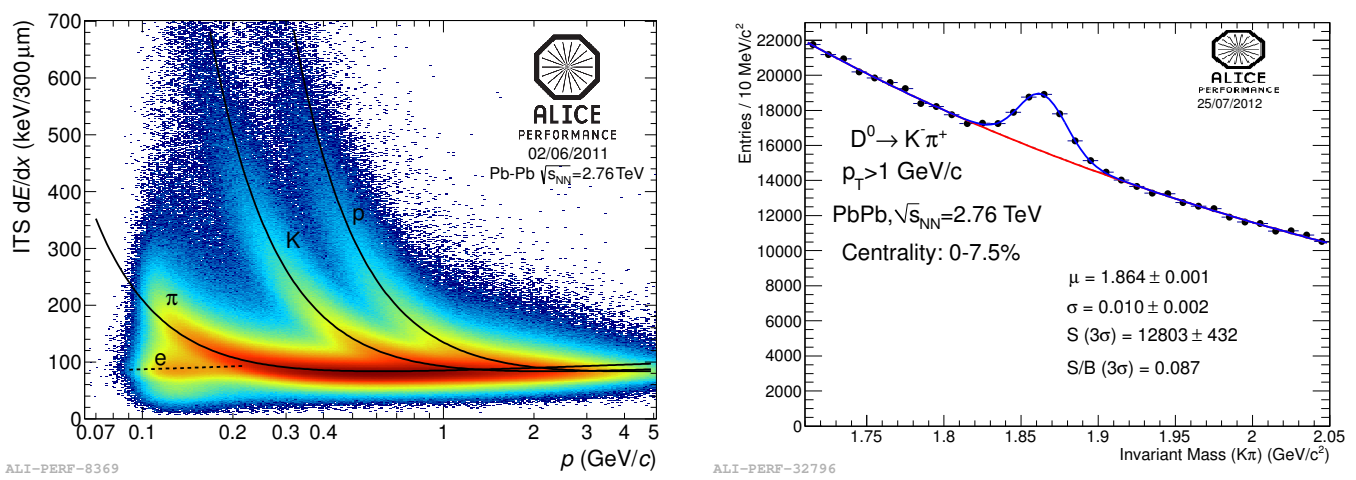

Figure 7: Left: $d E / d x$ for charged particles vs their momentum measured by the ITS in standalone mode in $\mathrm{Pb}-\mathrm{Pb}$ collisions at $2.76 \mathrm{TeV}$. Right: invariant mass distribution of $D^{0} \rightarrow K^{-} \pi^{+}$candidates for $p_{T}>1 \mathrm{GeV} / \mathrm{c}$ in $\mathrm{Pb}-\mathrm{Pb}$ collisions at $2.76 \mathrm{TeV}$.

the particle (pions, kaons and protons) spectra analysis at low $\mathrm{p}_{T}$. Furthermore due to its precise determination of the track impact parameter and to its reconstruction capability of the primary vertex the ITS is crucial for the physics study in the charm sector.

\section{References}

[1] ALICE collaboration, J. Phys. G: Nucl. Part. Phys.32, 2006, 1295.

[2] ALICE collaboration, JINST 3, 2008, S08002.

[3] ALICE Off-line framework, AliRoot, http://aliceinfo.cern.ch/Offline.

[4] Andrea Rossi, Ph.D. Thesis, Università degli Studi di Trieste, 2010.

[5] V. Blobel, Nucl. Inst. and Meth. A566, 2006, 5.

[6] ALICE collaboration 2010 JINST 5 P03003.

[7] C. Bombonati et al., ALICE Internal Note 2009-035 (2009).

[8] A. Dainese et al., ALICE Internal Note 2009-045 (2009).

[9] M.Lunardon for the ALICE collaboration, proceedings of PLHC10, DOI: http://dx.doi.org/10.3204/DESY-PROC-2010-01/192.

[10] E. Bruna, A. Dainese, M. Masera, F. Prino, ALICE-INT-2009-018, 2010.

[11] E. Crescio, A. Dainese, M. Masera, F. Prino, ALICE-INT 2009-046.

[12] X.Wang and M. Gyulassy, Phys. Rev. D44, 1991, 3501. 